\title{
Autonomous discovery of materials for intercalation electrodes
}

Bolle, Felix Tim; Mathiesen, Nicolai Rask; Juul Nielsen, Alexander; Vegge, Tejs; García Lastra, Juan Maria; Castelli, Ivano Eligio

Published in:

Batteries and Supercaps

Link to article, DOI:

10.1002/batt.201900152

Publication date:

2020

Document Version

Peer reviewed version

Link back to DTU Orbit

Citation (APA):

Bolle, F. T., Mathiesen, N. R., Juul Nielsen, A., Vegge, T., García Lastra, J. M., \& Castelli, I. E. (2020). Autonomous discovery of materials for intercalation electrodes. Batteries and Supercaps, 3(6), 488-498. https://doi.org/10.1002/batt.201900152

\section{General rights}

Copyright and moral rights for the publications made accessible in the public portal are retained by the authors and/or other copyright owners and it is a condition of accessing publications that users recognise and abide by the legal requirements associated with these rights.

- Users may download and print one copy of any publication from the public portal for the purpose of private study or research.

- You may not further distribute the material or use it for any profit-making activity or commercial gain

- You may freely distribute the URL identifying the publication in the public portal 


\section{BATTERIES \& SUPERCAPS}

\section{Accepted Article}

Title: Autonomous discovery of materials for intercalation electrodes

Authors: Felix Tim Bolle, Nicolai Rask Mathiesen, Alexander Juul Nielsen, Tejs Vegge, Juan Maria Garcia Lastra, and Ivano Eligio Castelli

This manuscript has been accepted after peer review and appears as an Accepted Article online prior to editing, proofing, and formal publication of the final Version of Record (VoR). This work is currently citable by using the Digital Object Identifier (DOI) given below. The VoR will be published online in Early View as soon as possible and may be different to this Accepted Article as a result of editing. Readers should obtain the VoR from the journal website shown below when it is published to ensure accuracy of information. The authors are responsible for the content of this Accepted Article.

To be cited as: Batteries \& Supercaps 10.1002/batt.201900152

Link to VoR: http://dx.doi.org/10.1002/batt.201900152

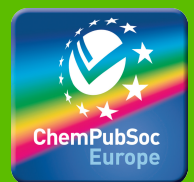




\title{
Autonomous discovery of materials for intercalation electrodes
}

\author{
Felix Tim Bölle * Nicolai Rask Mathiesen* Alexander J. Nielsen* \\ Prof. Tejs Vegge * Ass. Prof. Juan Maria García Lastra *
}

\author{
Asst. Prof. Ivano E. Castelli *†
}

\section{Graphical Abstract}

A workflow, in the framework of Density Functional Theory, has been designed and implemented that automatically calculates crucial battery properties. The automated calculations include the thermodynamic and mechanical stability, Open Circuit Voltages as well as kinetic barriers obtained through the Nudged Elastic Band method. The workflow was able to discover interesting candidates for magnesium insertion cathode materials without the need of user intervention.

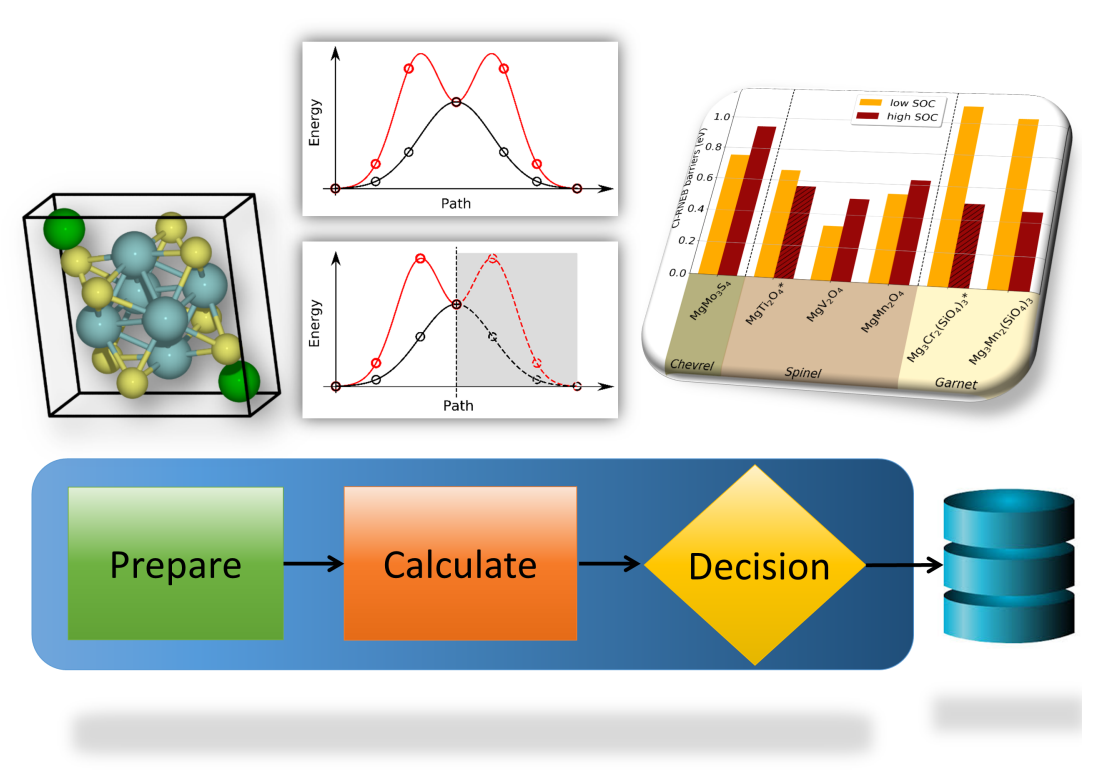

Figure 1: Graphical Abstract

\section{Abstract}

The development of automated computational tools is required to accelerate the discovery of novel battery materials. In this work, we design and implement a workflow, in the framework of Density Functional Theory, which autonomously identifies materials to be used as intercalation electrodes in batteries, based on descriptors like adsorption energies and diffusion barriers. A substantial acceleration for the calculations of the kinetic properties is obtained due to a recent implementation of the Nudged Elastic Bands (NEB) method, which takes into consideration the symmetries of the system to reduce the number of images to calculate. We have

\footnotetext{
* Department of Energy Conversion and Storage, Technical University of Denmark, Anker Engelunds Vej 411, Building 301, 2800 Kgs. Lyngby, Denmark.

$\dagger$ corresponding author - ivca@dtu.dk
} 
applied this workflow to discover new cathode materials for $\mathrm{Mg}$ batteries, where two of these materials display a threefold increase in the potential of the Chevrel phase, the state-of-the-art cathode in commercial prototype Mg batteries.

\section{Introduction}

The prospects of substantial global warming calls for a transition towards a more sustainable energy infrastructure, based on the production of energy from renewable sources. Energy storage devices, e.g. rechargeable batteries, play a fundamental role in the transition from fossil fuels to renewable energy sources, as highlighted from the recent (2019) Nobel price in Chemistry awarded to Yoshino, Whittingham, and Goodenough.

Nowadays, Li-ion is the prevalent technology in electric vehicles and even used for stationary storage applications. While the concomitant development of portable electronics and that of the Li-ion technology are apparent, the ability of battery technologies to meet the necessary requirements cannot be taken for granted in other areas. Aside concerns on availability and cost of lithium and other critical raw materials (CRM), some general arguments could be raised against a potential generalized implementation of lithium based technology at large scale. Amongst the most promising alternative to Li-chemistries are the Na-ion batteries and analogous technologies based on multivalent cations like $\mathrm{Mg}^{2+}, \mathrm{Ca}^{2+}, \mathrm{Zn}^{2+}$ and $\mathrm{Al}^{3+}$.[1]. The main drawback in $\mathrm{Mg}$, $\mathrm{Ca}, \mathrm{Zn}$ and $\mathrm{Al}$ batteries is related to the slow diffusion of multivalent elements, which drastically reduces the power performance. The foremost benefit is that for achieving a given capacity, the number of ions needed are reduced by a factor equal to the ion valence. This turns out in less stressed electrode materials and seemingly less degradation upon cycling. Multivalent electropositive metals include aluminum, zinc, calcium and magnesium. Aluminum, in spite of limited potential (2 V) and capacity (70 mAh/g) values.[2] Regarding calcium, initial studies show fast rate capability.[3] Arguably, the most interesting multivalent ion is magnesium, where significant progresses in performance has been achieved.[1] The state-of-the-art cathode in commercial prototype $\mathrm{Mg}$ batteries is the $\mathrm{Mo}_{6} \mathrm{~S}_{8}$ Chevrel phase, which is a covalent host where Coulombic interactions are reduced, favoring $\mathrm{Mg}$ ion diffusion. [4] However, the potential achievable from this cathode is rather low $(\approx 1 \mathrm{~V}$ vs $\left.\mathrm{Mg} / \mathrm{Mg}^{2+}\right)$. The discovery of novel cathode materials which can display higher potentials keeping, or even better improving, the ion diffusivity, is necessary to boost the Mg-ion technology.

Following an Edisionian "trial and error" approach to search for new materials is extremely time consuming and inefficient. During the last decades, quantum mechanical calculations, mostly within the framework of Density Functional Theory (DFT), have been efficiently used to predict properties and design novel materials for multiple applications. Recently, to accelerate the materials discovery, autonomous workflow schemes have been implemented, $[5,6,7,8,9]$ and more specifically for the discovery of battery materials, where a special focus was put on the kinetic properties, i.e. cation diffusivity.[10, 11] Including the kinetic aspect in an autonomous workflow is rather expensive and, in most cases, some approximations need to be done to compromise between accuracy and computational cost, e.g. pinball model[12] or bond-valence calculations.[11]

These workflows are usually based on generic tools that in addition to enable the complex inter-dependencies of the workflow steps allow for an easier reproducibility and provenance of the data [13, 14]. On one side, this helps researchers to share knowledge and expertise in the form of workflows, which can be reused by others as well as extended to include more features, and from the other they solve many issues[15] related with consistency and reproducibility of the data. Workflows, in fact, enable the creation of consistent data sets by setting all the parameters at the beginning of the project and being consistent with them.

Most of the design for new materials proceeds through a 'funnel/sieve scheme', where at each steps materials, which do not fulfill the desired properties, are discarded. In addition, the complexity of the calculations and their simulation cost increases at each step. Examples of funnel schemes and workflows for, e.g., visible solar light ferroelectric devices can be found in the literature.[16] In these cases, materials are selected for their stability, light harvesting properties, and interfaces. The discovery of battery materials is not dissimilar from these examples.

In this work, we establish an autonomous workflow to identify intercalation electrodes in batteries. Although we specifically search for novel cathodes for $\mathrm{Mg}$ batteries, the workflow is general enough to allow for the discovery of both cathode and anode electrodes for other battery technologies. In detail, the workflow is based on descriptors calculated at the DFT level, such as volume change upon charge/discharge, which gives information on the stability of the cathode, $\mathrm{Mg}$ adsorption energy, which indicates the open circuit voltage $(\mathrm{OCV})$, and diffusion barriers, which is a descriptor for the ion mobility at different charge states. For the first time in a workflow, the kinetic aspects are kept at the DFT level of accuracy.

\section{Workflow overview}

Figure 2 gives a schematic view of the seven main components of the workflow. The input from the user consists of choosing input parameters as well as providing a list of materials, i.e. compositions and structures, that will 
be investigated. Additionally, a database containing reference structures for the evaluation of the stability of an input structure on the convex hull is needed. The reference structures workflow is separated from the general workflow for finding ion-insertion cathode electrodes but ensures a consistent choice of input parameters for the reference calculations. Subsequently, the workflow autonomously takes care of generating and relaxing all needed structures necessary to determine crucial battery material properties. The three properties currently calculated are the stability (volume change upon charging/discharging and the thermodynamic stability), the electrode potential (Mg-adsorption energy) and the ion diffusivity (Mg-diffusion barriers). The two latter properties are evaluated both at a low and high state of charge (SOC). With respect to other work recently published [17], this workflow accelerates the calculations of the ion diffusivity by making use of symmetry considerations. In the following, the steps of the workflow are discussed in more detail. The workflow is interfaced with the Atomic Simulation Environment (ASE). [18] Moreover, it is intended to work with the VASP DFT code [19, 20, 21], as well as with the workflow management system MyQueue. [22] Efforts have been made to keep the workflow as general as possible in order to use the flexibility of ASE, which allows an interface with other DFT codes.

\subsection{User input}

In addition to the list of materials to investigate, the user needs to define 4 parameters:

- ion: The ion that will be studied which typically is an alkali or alkali-earth ion (i.e. Li, $\mathrm{Na}, \mathrm{K}, \mathrm{Mg}, \mathrm{or}$ $\mathrm{Ca})$.

- material ID: A unique identifier for each structure that is used to avoid duplicate calculations.

- magnetic state: The magnetic state parameter allows to study different magnetic starting configurations. This magnetic state is used for all structures throughout the workflow. The two options are the "nonmagnetic" initialization or to initialize all ions "ferromagnetically". The ferromagnetic initialization is carried out by initializing magnetic ions in a high spin state.

- Nudged Elastic Band (NEB) path length: The NEB path length determines the maximum linear distance between the initial and final site of the ion diffusing along the path. Paths that exceed this maximum length are neglected.

Three decision blocks are directly incorporated in the workflow scheme to decide whether to discard the candidate material or proceed with the calculations. The threshold values are given by the user deciding whether a material is suitable for further investigation or not, e.g. full computational OCV curve [23] or experimental validation. These thresholds are the Potential threshold, which sets a requirement on the minimum average OCV, and the Diffusivity threshold, which favors the search for materials exhibiting low diffusion barriers (Figure 2). A Stability threshold can be given optionally by setting constraints on the maximum volume change upon discharging or the energy above the convex hull for the fully charged/discharged structures.

\subsection{Calculation steps}

The potential materials investigated in the screening presented here are obtained from computational and experimental databases, such as the Materials Project,[24] the Open Quantum Materials Database (OQMD),[25] and Inorganic Crystal Structure Database (ICSD).[26] All these materials have in common that the charge carrier (from now on, we explicitly write the charge carrier as $\mathrm{Mg}$, although the workflow is not limited to identify cathode materials for $\mathrm{Mg}$ batteries) is present in the structure. Therefore, the starting structure of the workflow is always a fully discharged cathode material, from which we are going to remove magnesium. The workflow presented here is, however, more general and it could also be used to discover cathodes where the charge carriers are not present and intercalate in the interstitial sites.

\subsubsection{Structure optimization}

The first step is to relax the input structure (with all Mg-ions, fully discharged state); the calculation details are discussed in Section 7. In parallel, we calculate the same structure where we have removed all Mg-ions (deionized unit cell, optimized charged unit cell), as indicated in Figure 3. Both relaxations are carried out by the cell shape, volume and the atomic positions. From these two relaxations, it is possible to calculate the volume change in the charged and discharged states, which gives an indication on the mechanical stability of the battery. In addition, we relax a charged unit cell constraining its cell shape and volume. This is done by removing all $\mathrm{Mg}$ ions from the optimized discharged unit cell and scaling all atomic positions and the cell according to the ratio between the volume of the optimized charged unit cell and the optimized discharged unit cell, $r=V_{\text {charged }} / V_{\text {discharged. }}$ Subsequently, all atomic positions are relaxed while keeping the cell shape and volume fixed. This structure, referred to as discharged-constraint unit cell, is used for the calculation of the OCV at a high SOC as it is explained in more detail in Section 4.2.5. 


\subsubsection{Decision point 1 - Stability}

The volume change is calculated as

$$
\Delta V=\frac{V_{\text {charged }}-V_{\text {discharged }}}{V_{\text {discharged }}}
$$

where $V_{\text {charged,discharged }}$ are the volumes of the unit cell with/without $\mathrm{Mg}$-ions, respectively. The energy above the convex hull $E_{\text {hull }}$ is evaluated for both structures giving an indication on the thermodynamic stability of the fully charged and discharged structures. Large energies above the hull will have implications on the structures cyclability and synthesizability and might lead to unwanted conversion reactions. [27] On the other hand, due to kinetic stabilization metastable structures can still lead to the discovery of viable electrodes which makes it difficult to define a threshold value upfront.[28, 29]

\subsubsection{Preparation of the structures with Mg-vacancies}

To limit possible errors associated with defect-defect interactions, we need to create a supercell, which is obtained by repeating the relaxed unit cell from 4.2.1 until it satisfies the condition that the spacing between two vacancies is at least twice the NEB path length, defined in the user input. To ensure relatively fast calculations, we have added an additional requirement on the number of atoms in the supercell, which should not exceed 200 atoms. If this limit is reached, the workflow will automatically reduce the NEB path length (vacancy spacing) parameter to ensure that the maximum number of atoms in the supercell is not exceeded. To save computational time and retain symmetries, we assume that the supercell is fully relaxed after its creation. Subsequently, each of the symmetry-in-equivalent $\mathrm{Mg}$ ions is removed one-by-one from the supercell structure. The newly created defected structures, with one $\mathrm{Mg}$ vacancy, are analyzed by the Symmetry Equivalence Check tool, as implemented in ASE. The tool is based on the recipe as explained in Ref. [30]. Symmetry in-equivalent sites are checked for percolating paths. Possible diffusion paths are only accepted if intermediate images obtained through linear initialization between initial and final images do not get closer than $1 \AA$ to any atoms in the host structure. If combining all possible diffusion paths does not lead to percolating paths, i.e. the ion cannot traverse through the full structure, the selected ion occupying the site is non-conductive. In such cases the structure is discarded.

Once that the symmetry in-equivalent sites have been identified, a similar procedure is used to determine the symmetry in-equivalent NEB paths by removing the initial and final $\mathrm{Mg}$ site of the path from the structure. Checking for symmetry in-equivalent vacancy defect pairs (possible NEB paths) at this point of the workflow allows us to rank the materials according to the number of NEB calculations which need to be run. The Symmetry Equivalence Check tool identifies all the in-equivalent NEB paths that can be drawn between two Mg ions, which are spatially apart by less than the NEB path length value.

The output of this step of the workflow therefore consists not only of the supercell, but also of the information on the symmetry of the structure with respect to $\mathrm{Mg}$-vacancies, which practically is a list of which $\mathrm{Mg}$ (or vacancy defect pairs) are symmetrically equivalent. Before continuing with the (heavy) optimization of the supercell, it is therefore possible to get a rough estimate of the number of NEB calculations that need to be performed to evaluate the size of the diffusion barrier. The NEB paths are also checked for reflection symmetry at this point. Reflection symmetry will result in a less computationally expensive NEB calculation as will be explained in 4.2 .6 .

One problem that is frequently encountered is the correct labelling of defect sites. Indices of structures change as soon as vacancy defects are created. In order to work around this issue we tried to work with scaled positions of defects instead of specific indices whenever possible. If indices for specific sites were used (i.e. initial and final site in the NEB paths) they always match the indices in the created supercell.

\subsubsection{Optimization of the vacancy defect structures}

The optimization of the vacancy defect structures is carried out for the dilute vacancy limit (low SOC) as well as for the high vacancy limit (high SOC). For the dilute vacancy limit, a single Mg-vacancy is created by removing only the symmetry in-equivalent ions from the supercell. In this case, the cell shape and volume is kept fixed during the relaxation and only the atomic positions are optimized. In the limit of high vacancy concentration, only a single symmetry in-equivalent $\mathrm{Mg}$ is left in the supercell and the supercell is scaled according to the ratio between the volume of the optimized charged unit cell and the optimized discharged unit cell, $r=V_{\text {charged }} / V_{\text {discharged }}$ (see also Figure 3 ). Therefore, in the limit of high vacancy concentration, the shape and the volume of the supercell is fixed during relaxation and all atomic positions as well as lattice parameters are scaled. By keeping the cell shape fixed, the symmetries from the dilute vacancy limit case are 
preserved and the reflection symmetries have not changed. This enables to use the advantage of a reflection symmetry (reflective NEB) during the computationally expensive NEB calculations, as explained in 4.2.6. The assumption of a fixed cell shape even for the low $\mathrm{Mg}$ concentration limit will not hold in situations where a rather drastic change in cell shape upon charging occurs. On the other side, such materials would most likely break all contacts in a real battery and it should probably be discarded [31], irrespective. This could be checked already during step 4.2.1.

\subsubsection{Decision point 2 - Minimum voltage}

The calculations performed so far allow us to calculate the potential at different SOCs, which is a descriptor for the minimum voltage of the cathode electrode. The different charge states include the low SOC (removing a single $\mathrm{Mg}$ from the supercell), calculated as

$$
\mathrm{V}_{\text {low }-\mathrm{SOC}}=\frac{\left(\mathrm{E}_{\text {low }-\mathrm{SOC}}+\mathrm{E}_{\mathrm{bulk}-\mathrm{Mg}}\right)-\mathrm{E}_{\text {discharged }}}{\mathrm{z}}
$$

where $E_{\text {discharged }}$ is the DFT energy of the relaxed unit cell, $E_{\text {low-SOC }}$ is the DFT energy of the supercell in the dilute vacancy limit, $E_{b u l k-M g}$ is the DFT energy of a single Mg atom in its most stable bulk structure and $z$ is the amount of electrons transferred (in the case of $\mathrm{Mg}, z=2$ ), the high SOC (removing all but one ion from the supercell) calculated as

$$
\mathrm{V}_{\text {high-SOC }}=\frac{\left(\mathrm{E}_{\text {charged-constrained }}+\mathrm{E}_{\mathrm{bulk}-\mathrm{Mg}}\right)-\mathrm{E}_{\mathrm{high}-\mathrm{SOC}}}{\mathrm{Z}}
$$

where $E_{\text {high-SOC }}$ is the DFT energy of the supercell in the high vacancy limit and $E_{\text {charged-constrained }}$ is the DFT energy of the deionized unit cell with the same cell shape as the supercell in the high vacancy limit but with a scaled volume. The last charge state considered is the average OCV calculated as

$$
\mathrm{V}_{\text {average }}=\frac{\left(\mathrm{E}_{\text {charged }}+\mathrm{n} \cdot \mathrm{E}_{\mathrm{bulk}-\mathrm{Mg}}\right)-\mathrm{E}_{\text {discharged }}}{\mathrm{n} \cdot \mathrm{z}}
$$

where $E_{\text {charged }}$ is the DFT energy of the deionized unit cell and $n$ is the amount of $\mathrm{Mg}$ ions in the discharged supercell. $^{1}$

\subsubsection{Preparation of NEB paths and calculation of diffusion barriers}

Methodologies to accelerate NEB calculations have been discussed in the literature. For instance, Rong et al. [32] make use of an improved initial path guess over the usual linear initialization scheme and subsequently perform single point calculations on the images. They predict diffusion barriers that are within $20 \mathrm{meV}$ compared to DFT calculations for Li systems. A different approach probes the potential energy surface (PES) using a gaussian process regression model to find the minimum energy path $[33,34]$. In this work, we investigate the possibility of using the recently implemented Reflective - Nudged Elastic Band Method (R-NEB) for accelerating NEB calculations (see [17] for details about the implementation). In contrast to the two other methods, this does not approximate the PES but rather accelerates NEB calculations by considering symmetry operations to reduce the images to calculate.

The logic behind the preparation of the NEB path, in fact, decides which kind of NEB calculation to perform, as schematized in Figure 4 (a). All NEBs are run with ASEs internal NEB optimizer. We initialize the magnetic moments of all images using the information from the previously relaxed initial and final images. The total magnetic moment is forced to be the same in the initial and intermediate images. If no convergence is observed we remove the constraint of a fixed total magnetic moment and carefully inspect the structure visually after the workflow finishes for consistent magnetic moments.

The step 4.2.3 provides the necessary information about all the defect structures and their symmetries. A NEB path can be drawn by taking two of these structures, which are indicated as initial and final image. In case of the path not being reflective or the initial and final image not being symmetry equivalent, a conventional NEB is performed. We define a conventional NEB calculation as the process of setting up the initial and final structures followed by two separate relaxations of the initial and final image. After this, intermediate images, built through an interpolation of the initial and final relaxed structures, are added to the full path length (Figure $4(\mathrm{~b}))$.

If the initial and final image are symmetry equivalent the NEB path is tested for reflection symmetry. In three

\footnotetext{
${ }^{1}$ Note: These expressions are valid only if the energies are expressed in $\mathrm{eV}$.
} 
dimensions, the reflection symmetries can be reflective with respect to a plane (reflecting on one mirror plane), line (reflecting on two mirror planes), or point (reflecting on three mirror planes). If at least one reflection symmetry is found, we can perform a reflective-middle-image NEB (RMI-NEB) calculation. The RMI-NEB (sketched in Figure 4 (d)) is closely related to the Reflective NEB (R-NEB) method (sketched in Figure 4 (c)), which is also explained in ref. [17]. In contrast to a conventional NEB where initial and final image are supplied, RMI-NEB needs only the initial image, as the final image can be created through symmetry operations from the initial image. This is schematically shown in Figure 4 (d) where the final image lies in the grey shaded area (dashed circle) indicating that the image has been created by symmetry operations rather than from a full DFT relaxation. Only one single image (middle image) needs to be calculated now. With respect to a conventional NEB run with a single middle image, this improvement and speed-up here is that the relaxation of the final image is not needed. This speed-up is even more if several images are used for the NEB path. Consider a NEB path with five intermediate images (Figure 4 (b)). The reflective NEB (Figure 4 (c)) only considers three images including two intermediate images as well as the middle image. The two remaining intermediate images are then created by symmetry operations.

Using the approach of a single middle image, we always find an energy barrier that is smaller or equal to the energy of the transition state. For instance, it finds the correct transition state energy in case of a truly bellshaped barrier (Figure 4 (d) solid black line) but it does not return the true height of the energy barrier in case of an energy barrier that has two maxima. Figure 4 (d) shows the true energy barrier curve with two maxima (grey line) as well as the found energy barrier curve with the RMI-NEB method in red that overlaps with the truly bellshaped energy barrier. An energy barrier with two maxima is rather common and can for example be found for the diffusion of $\mathrm{Mg}$ in Spinel structures [35], where the middle image corresponds to a configuration of the $\mathrm{Mg}$ ion in an octahedral site. In order to find the global maximum and the true height of the energy barrier in such example cases, we can subsequently run a climbing image reflective NEB (CIRNEB), if the barrier is not already above the diffusion threshold value. Reflection symmetry is again applied by mirroring half of the intermediate images. The climbing image now finds the exact energy of the transition state for a barrier shape with two maxima (Figure 4 (e) solid red line). We stop the CI-RNEB in case we find all intermediate images to lie below the extracted maximum energy from the RMI-NEB, because this indicates that the maximum energy barrier has already been found (black line, Figure 4 (e)).

We note that the initialization of the NEB paths is done by linear interpolation of two images. However, it is possible to follow different path initialization schemes, which might be preferred, especially when running a conventional NEB. For instance, the image dependent pair potential method providing an improved initial guess for the NEB path is already implemented in ASE requiring minimal changes in the code. In case of the reflective NEB paths, the user would have to make sure that the initialized path preserves the reflection symmetry which is not implemented in the presented workflow.

\subsubsection{Decision point 3 - ion diffusivity and data collection}

The final step of the workflow is to calculate the Mg diffusivity, which can be estimated from the Mg diffusion barriers obtained from the NEB method.

At the end, all the structures, calculations, and estimated properties are collected and stored in a database.

\subsection{Analysis of the workflow}

The workflow described here can be divided into three different layers, as indicated in Figure 5. The bottom layer contains scripts that directly interact with the calculation engine (VASP in this case) or other tools used to prepare and optimize the structures (ASE). Moreover, this layer includes all parameters used during the calculations, which are strictly connected with the simulation packages used. This layer is the least generic of the workflow, because it depends on the choice of code and parameters, decided by the user. This means that the middle layer of the workflow, or workflow layer, which contains the building blocks of the workflow, should allow the user to change any of the blocks of the bottom layer, e.g. the DFT code, without damaging all of the workflow structure. The workflow layer can be structured in three different blocks: a prepare block that prepares the structure to calculate and includes the logic to extract the symmetry equivalent structures; an optimize block that directly accesses the simulation tools and setups to optimize the various structures; and a decision block that acts at the decision points of the workflow and decides whether a material should be considered as a potential candidate for further calculations. The top layer consists of the workflow management system as well as the script to collect the generated data. Various workflow management systems have been implemented to be used in connection with materials science (e.g. AiiDA [13] or Fireworks [14]). In our case, we use an in-house tool called MyQueue [22], which deals with all the operations to submit (and resubmit failed) calculations as well as setting up the dependencies between the different steps of the workflow. Additionally, 
we included specific instructions on how to restart automatically failed or timed out calculations throughout the workflow similar to the CUSTODIAN package as implemented in Pymatgen [36].

\section{Practical Application: Cathode Materials for Mg-ion Batteries}

We used this workflow to find novel candidate materials to be used as cathodes in Mg-ion batteries. As the aim of this work is to describe the implementation of the workflow, we show, as a proof of concept, only a small subset of the possible materials that can be calculated.

$\mathrm{Mo}_{6} \mathrm{~S}_{8}$ in the Chevrel phase $\left(\mathrm{MgMo}_{3} \mathrm{~S}_{4}\right.$, shown in Fig. 6 (a)) is currently the state-of-the-art for commercial prototype $\mathrm{Mg}$ cathodes as it can accommodate $\mathrm{Mg}^{2+}$-ions for hundreds of cycles at stable potentials and charge capacities. [4] This phase is chosen as the benchmark material and its diffusion and OCV properties provide the necessary input thresholds needed for the workflow.

\section{Initial pool of materials to investigate}

We investigate only ternary and quaternary structures containing a transition metal, $\mathrm{Mg}$ and O. These structures are taken from the ICSD database.[26] This choice is made to speed-up the synthesis of the identified materials as their synthetic pathway is established. Prior to running first principle investigations we apply filters that do not require the execution of DFT calculations. Metrics and design rules for finding fast magnesium conducting materials have been discussed extensively in literature (e.g. [37, 38]). Based on these design rules, we only study materials where $\mathrm{Mg}$ is not in its preferred anion coordination environment in the interstitial site as large diffusion energy barriers are expected. The preferred anion coordination environment for a $M g^{2+}$ ion in crystallography literature has been determined to be six-fold.[39] The coordination number of anions is determined using a Voronoi Tessellation of all atoms followed by discarding all anion neighbors that are more than 1.2 times the expected covalent ion-anion bond distance away from the $\mathrm{Mg}$ atom. In addition to the coordination number filter, only the structures without partial occupancy are considered. If the oxidation state of the transition metal inside the structure is exceeded upon fully charging the cathode material, the structure is excluded from the pool of starting candidates. A third filter criterion ensures that at least one percolating path for Mg exists in the structure prior to running the calculation. The structure is discarded in case all Mg ions are trapped. Less than 25 ternary and quaternary materials fulfill these criteria. In the present work, we consider a subset of 5 structures in addition to the benchmark material, which show reflection symmetry for the NEB paths. The benchmark material was taken from the Materials Project database [24] due to the partial occupancy of magnesium in the structure from the ICSD database. All considered structures comprise three different crystal groups which are shown in Figure 6.

\subsection{Results}

The discharged structures in this study are known to exist and therefore show energies below 0.1 eV above the hull (Fig. 7). For the charged structures especially the silicates exhibit larger energies above the hull of more than $0.4 \mathrm{eV}$. This might indicate poor stability upon charging the battery using this type of cathode as unwanted conversion reactions might occur. As expected, all materials show the tendency to reduce their volumes upon charging ( $\mathrm{Mg}$ is removed), as shown in Figure 8 (a). The Manganese Spinel structure $\left(\mathrm{Mg}_{2} \mathrm{Mn}_{4} \mathrm{O}_{8}, \mathrm{Figure} 6\right.$ (c)) shows the largest distortion among the studied materials. This has been already discussed in literature and has been referred to the filling of higher energy anti-bonding $\mathrm{e}_{g}$ orbitals for Mn upon reduction as the lower energy $\mathrm{t}_{2 g}$ anti-bonding orbitals are fully occupied leading to larger volume changes (this is not the case for other elements, such as Ti, for which free lower energy $t_{2 g}$ orbitals can be occupied). [35, 40]

The OCVs indicate that all materials have a higher average OCV than the Chevrel phase (Figure 8 (b)). The voltages lie in between a range of $1.3-4.5 \mathrm{~V}$ making these materials interesting as $\mathrm{Mg}$ cathodes as the OCV is higher than the benchmark material. The only exception is $\mathrm{MgTi}_{2} \mathrm{O}_{4}$ (Fig. 8 (b)), which has a calculated voltage below the average voltage of the Chevrel phase at a low SOC. Since all materials fulfill the first requirement (average OCV larger than the OCV for the Chevrel phase), we proceed with investigating the Mg-diffusion using NEBs.

The barriers calculated for the benchmark material $\mathrm{MgMo}_{3} \mathrm{~S}_{4}$ are $\sim 750 \mathrm{meV}$ at a low SOC and $\sim 940$ $\mathrm{meV}$ at a high SOC (Figure $9 \mathrm{a}+\mathrm{b}$ ). They suggest that the multivalent $\mathrm{Mg}^{2+}$-ions are more mobile at a low SOC, i.e. a structure with a high concentration of $\mathrm{Mg}^{2+}$. The Chevrel phase is the only material that has its transition state barrier at the middle image for the high and low SOC structures. This means that in this case evaluating the barriers using the RMI-NEB method results in the same barriers as obtained using the CI-RNEB method. The Spinel structures $\left.\left(\mathrm{MgTM}_{2} \mathrm{O}_{4}, \mathrm{TM}=\{\mathrm{Ti}, \mathrm{V}, \mathrm{Mn}\}\right)\right)$ show low transition state barriers at the middle images $(\sim 30-420 \mathrm{meV})$. Higher mobility is again observed at a low SOC in agreement with what has 
been found in recent NEB studies on Spinel structures (e.g. $\mathrm{MgMn}_{2} \mathrm{O}_{4}$ in [35]). Although these barriers are significantly lower than the ones for the benchmark material, the transition state is not found at the middle image which becomes obvious when looking at barriers obtained using a climbing image in Fig 9 b $(\sim 320-660$ $\mathrm{meV})$. NEB calculations reported in the literature indicate a barrier shape with two maxima, as can be seen in Ref. [35] The RMI-NEB method thus fails to find such a global maximum (as described in details above, Figure 4 (d)). The reason behind this shape of the barrier is the diffusion topology, which results in the $\mathrm{Mg}^{2+}$-ion diffusing from an initial tetrahedral site through an octahedral intermediate coordination to its final site which is also tetrahedral. In the case of $\mathrm{MgTi}_{2} \mathrm{O}_{4}$, the intermediate octahedral site has a lower energy than the initial and final tetrahedral site. This is indicated by formulas marked with an asterisk and the hatched bar in Figure 9. This suggests that the pathway should start from the octahedral site and not from the tetrahedral sites [35]. Experimental studies on the cubic thiospinel structure $\mathrm{Ti}_{2} \mathrm{~S}_{4}$ find partial occupancies of $\mathrm{Mg}$ in octahedral and tetrahedral sites.[41] These findings might also apply to the oxide spinel $\mathrm{Ti}_{2} \mathrm{O}_{4}$ studied here.

The two silicate structures $\left.\left(\mathrm{Mg}_{3} \mathrm{TM}\left(\mathrm{SiO}_{4}\right)_{3}, \mathrm{TM}=\{\mathrm{Cr}, \mathrm{Mn}\}\right)\right)$ have low transition state barriers at the middle images for a high SOC (below $\sim 100 \mathrm{meV}$, where the middle image of $\mathrm{Mg}_{3} \mathrm{Cr}\left(\mathrm{SiO}_{4}\right)_{3}$ is again lower in energy than the initial image). The barriers found using the CI-RNEB are again larger, indicating the transition state does not coincide with the middle image of the path. The diffusion is significantly slower at a low SOC, as shown by the higher transition state energy barrier above $1 \mathrm{eV}$. This is similar to olivine structures studied in the literature $\left(\mathrm{FePO}_{4}\right.$ [37], which also show larger diffusion barriers for moving multivalent ions at a low SOC compared to the diffusion barriers at a high SOC). By investigating the middle image from the RMI-NEB calculation and comparing the middle image at a low and high SOC, we note that at a high SOC, less $M g^{2+}$-ions compete to attract the negatively charged $\mathrm{O}^{2-}$-anions. The $\mathrm{Mg}$-O bonds between the diffusing $M g^{2+}$-ion and its closest oxygen neighbors are approximately $10 \%$ shorter for the middle image in the high SOC, compared to the low SOC case, which leads to a more stabilized structure and therefore a lower barrier. The lower transition state barrier might also be a result of the rather unstable charged phase of the silicates ( $\mathrm{E}_{\text {hull }}$ in Fig. 7). A more detailed study on the stability and possible conversion reactions is probably needed.

Within the same crystal symmetries, the materials show similar trends for the height of the diffusion barriers: the Garnet structures show a significantly slower diffusion of $\mathrm{Mg}^{2+}$ upon discharging whereas the diffusion barriers of the Spinels are much closer in energy for the high and low SOCs. This indicates that calculating diffusion barriers (and their shapes) for a few representative structures from each crystal group can be a useful approach to accelerate the materials discovery. These trends can be used to select the most promising crystal structures to study using the workflow proposed here. The energy barriers for a single diffusing Mg-ion in the studied oxide systems are thus governed by the diffusion path, which is specific for each crystal symmetry, as already reported in the literature.[37] In contrast, the OCV and volume changes are governed by the Red-Ox properties of the transition metal.

\section{Conclusions and Perspective}

We have presented a workflow based on DFT calculations, which autonomously identifies candidate materials to be used as intercalation electrodes in batteries. The workflow is composed of different steps, where the volume change and thermodynamic stability upon charging, the OCV at different charge states and the NEB diffusion energy barrier at high and low SOC are calculated. This workflow has been tested to identify possible cathode materials for $\mathrm{Mg}$ batteries, which can improve the performance (faster $\mathrm{Mg}^{2+}$ diffusion and higher OCVs) of the Chevrel phase; the most common cathode material in $\mathrm{Mg}$ batteries. In addition to identifying candidate materials, the workflow can provide quick insights, useful to investigate trends that can even further accelerate the discovery of novel materials. The workflow is continuously developed and may be accessed at https://gitlab.com/asc-dtu/workflows/ion-insertion-battery-workflow. The structures presented in this work together with the version of the workflow used here is available on FigShare [42].

We would like to point out that the workflow is a useful tool for an autonomous screening approach that gives insights in diffusion properties of materials, since calculating diffusivities is typically the most expensive part computationally, as well as manually going through and setting up the calculations. The workflow is not a universal tool for studying any cathode material and so far it only works for materials that have the cation inside the structure. We are currently working on extending it to include the following features:

- The workflow is not able to find the correct magnetic state ordering for all structures. Initializing all magnetic ions in a high spin state lead to satisfactory convergence for the materials shown but also failed to find the correct spin state for specific cases. We are currently working on finding a more automated way to find the correct magnetic ordering leading to the minimum energy configuration.

- Until now we only removed Mg ions from a structure that had the ion already inserted. It is in principle also possible to include structures where $\mathrm{Mg}$ is added into the interstitial sites and feed that structure to 
the workflow presented here. This would enable us to study crystal systems like 2-D layered structures without making changes to the workflow.

\section{Computational Details}

The computational details refer to the example described here in section 5, and can easily be modified. All calculations have been performed using the Vienna Ab-initio Simulation Package (VASP) using a plane-wave basis set.[19, 20, 21] The exchange and correlation effects are approximated using the Perdew-Burke-Ernzerhof revised for solids (PBEsol) functional.[43]. An energy cut-off of $520 \mathrm{eV}$ was applied to describe the valence electrons. Sampling the Brillouin zone is done using a k-point density $>4.7 \AA^{-1}$. As a required convergence criterion all forces on the movable nuclei had to be less than $0.03 \mathrm{eV} / \AA$. To account for the self interaction error in the $d$ orbitals of the transition metal oxides a $+\mathrm{U}$ correction is applied with the values taken from the Material Project database, i.e. $\mathrm{U}_{V}=3.25 \mathrm{eV}, \mathrm{U}_{C r}=3.7 \mathrm{eV}, \mathrm{U}_{M n}=3.9 \mathrm{eV}$.[24, 44] In the calculation for the convex hull, the reference for oxygen in the gas phase is approximated using the approach suggested by Rossmeisl et al.,[45] where the energy of oxygen at $0 \mathrm{~K}$ is calculated as the difference in energy of water and hydrogen molecules in gas phase, including the zero point energy (ZPE) corrections.

All NEB simulations [46] have been calculated using the GGA-PBEsol functional. The R-NEB method was applied [17] as implemented in the Atomic Simulation Environment package (ASE) [18], whenever reflection symmetry in the path is observed. As a required convergence criterion all forces had to be less than $0.05 \mathrm{eV} / \AA$. The $+U$ correction penalizes the energy of the transition state since it is a less localized state than the initial and final state. Thus, we do not apply a $+\mathrm{U}$ correction to the NEB calculation as also discussed in Ref. [35]. The distance between repeating defects in the super cells is always larger than $9 \stackrel{\AA}{A}$ to avoid defect-defect interaction errors.

\section{Acknowledgment}

The authors wish to acknowledge support from the European Magnesium Interactive Battery Community (eMagic) FET-Proactive project (Contract N. 824066). FTB, TV, IEC acknowledge support from the Department of Energy Conversion and Storage, Technical University of Denmark, through the Special Competence Initiative Autonomous Materials Discovery (AiMade).[47] NRM and JMGL acknowledge support from the Villum Foundation's Young Investigator Programme (4th round, project: In silico design of efficient materials for next generation batteries. Grant number: 10096) .

\section{Keywords}

Mg-Batteries, Autonomous discovery, workflow, Cathodes, DFT

\section{References}

\section{References}

[1] J. Muldoon, C. B. Bucur, and T. Gregory, "Quest for nonaqueous multivalent secondary batteries: Magnesium and beyond," Chemical Reviews, vol. 114, no. 23, pp. 11683-11720, 2014. PMID: 25343313.

[2] S. K. Das, S. Mahapatra, and H. Lahan, "Aluminium-ion batteries: developments and challenges," J. Mater. Chem. A, vol. 5, pp. 6347-6367, 2017.

[3] A. Ponrouch and M. Palacin, "On the road toward calcium-based batteries," Curr. Opin. Electrochem., vol. 9, pp. $1-7,2018$.

[4] A. Mitelman, M. Levi, E. Lancry, E. Levi, and D. Aurbach, "New cathode materials for rechargeable mg batteries: fast $\mathrm{mg}$ ion transport and reversible copper extrusion in cu y mo 6 s 8 compounds," Chemical Communications, no. 41, pp. 4212-4214, 2007.

[5] K. Tran, A. Palizhati, S. Back, and Z. W. Ulissi, "Dynamic workflows for routine materials discovery in surface science," Journal of chemical information and modeling, vol. 58, no. 12, pp. 2392-2400, 2018.

[6] J. H. Montoya and K. A. Persson, "A high-throughput framework for determining adsorption energies on solid surfaces," npj Comput. Mater., vol. 3, no. 1, p. 14, 2017. 
[7] S. Haastrup, M. Strange, M. Pandey, T. Deilmann, P. S. Schmidt, N. F. Hinsche, M. N. Gjerding, D. Torelli, P. M. Larsen, A. C. Riis-Jensen, et al., "The computational 2d materials database: highthroughput modeling and discovery of atomically thin crystals," 2D Materials, vol. 5, no. 4, p. 042002 , 2018.

[8] N. Mounet, M. Gibertini, P. Schwaller, D. Campi, A. Merkys, A. Marrazzo, T. Sohier, I. E. Castelli, A. Cepellotti, G. Pizzi, et al., "Two-dimensional materials from high-throughput computational exfoliation of experimentally known compounds," Nat. Nanotechnol., vol. 13, no. 3, p. 246, 2018.

[9] K. Kuhar, A. Crovetto, M. Pandey, K. S. Thygesen, B. Seger, P. C. K. Vesborg, O. Hansen, I. Chorkendorff, and K. W. Jacobsen, "Sulfide perovskites for solar energy conversion applications: computational screening and synthesis of the selected compound LaYS3," Energy Environ. Sci., vol. 10, no. 12, pp. 2579-2593, 2017.

[10] L. Kahle, A. Marcolongo, and N. Marzari, "High-throughput computational screening for solid-state li-ion conductors," arXiv preprint arXiv:1909.00623, 2019.

[11] N. A. Katcho, J. Carrete, M. Reynaud, G. Rousse, M. Casas-Cabanas, N. Mingo, J. RodríguezCarvajal, and J. Carrasco, "An investigation of the structural properties of li and na fast ion conductors using high-throughput bond-valence calculations and machine learning," J. Appl. Crystallogr., vol. 52, pp. 148-157, Feb. 2019.

[12] L. Kahle, A. Marcolongo, and N. Marzari, "Modeling lithium-ion solid-state electrolytes with a pinball model," Physical Review Materials, vol. 2, p. 065405, Jun 2018.

[13] G. Pizzi, A. Cepellotti, R. Sabatini, N. Marzari, and B. Kozinsky, "Aiida: automated interactive infrastructure and database for computational science," Computational Materials Science, vol. 111, pp. 218-230, 2016.

[14] A. Jain, S. P. Ong, W. Chen, B. Medasani, X. Qu, M. Kocher, M. Brafman, G. Petretto, G.-M. Rignanese, G. Hautier, et al., "Fireworks: a dynamic workflow system designed for high-throughput applications," Concurrency and Computation: Practice and Experience, vol. 27, no. 17, pp. 5037-5059, 2015.

[15] L. M. Ghiringhelli, C. Carbogno, S. Levchenko, F. Mohamed, G. Huhs, M. Lüders, M. Oliveira, and M. Scheffler, "Towards efficient data exchange and sharing for big-data driven materials science: metadata and data formats," npj Comput. Mater., vol. 3, no. 1, p. 46, 2017.

[16] I. E. Castelli, T. Olsen, and Y. Chen, "Towards photoferroic materials by design: Recent progresses and perspective," Journal of Physics: Energy, Sept. 2019.

[17] N. R. Mathiesen, H. Jonsson, T. Vegge, and J. M. Garcia Lastra, "R-neb: Accelerated nudged elastic band calculations by use of reflection symmetry," J. Chem. Theory Comput., vol. 15, no. 5, pp. 3215$3222,2019$.

[18] A. H. Larsen, J. J. Mortensen, J. Blomqvist, I. E. Castelli, R. Christensen, M. Dułak, J. Friis, M. N. Groves, B. Hammer, C. Hargus, et al., "The atomic simulation environmenta python library for working with atoms," Journal of Physics: Condensed Matter, vol. 29, no. 27, p. 273002, 2017.

[19] G. Kresse and J. Furthmüller, "Efficient iterative schemes for ab initio total-energy calculations using a plane-wave basis set," Phys. Rev. B, vol. 54, no. 16, p. 11169, 1996.

[20] G. Kresse and D. Joubert, "From ultrasoft pseudopotentials to the projector augmented-wave method," Phys. Rev. B, vol. 59, no. 3, p. 1758, 1999.

[21] P. E. Blöchl, "Projector augmented-wave method," Phys. Rev. B, vol. 50, no. 24, p. $17953,1994$.

[22] "Myqueue." https://myqueue.readthedocs.io.

[23] Y. S. Meng and M. E. Arroyo-de Dompablo, "First principles computational materials design for energy storage materials in lithium ion batteries," Energy Environ. Sci., vol. 2, no. 6, pp. 589-609, 2009.

[24] A. Jain, S. P. Ong, G. Hautier, W. Chen, W. D. Richards, S. Dacek, S. Cholia, D. Gunter, D. Skinner, G. Ceder, et al., "Commentary: The materials project: A materials genome approach to accelerating materials innovation," APL Mater., vol. 1, no. 1, p. 011002, 2013.

[25] J. E. Saal, S. Kirklin, M. Aykol, B. Meredig, and C. Wolverton, "Materials design and discovery with high-throughput density functional theory: the open quantum materials database (oqmd)," JOM, vol. 65, no. 11, pp. 1501-1509, 2013.

[26] "Inorganic crystal structure database (icsd)." http://www.fiz-karlsruhe.com/icsd.html.

[27] P. Canepa, G. Sai Gautam, D. C. Hannah, R. Malik, M. Liu, K. G. Gallagher, K. A. Persson, and G. Ceder, "Odyssey of multivalent cathode materials: open questions and future challenges," Chemical reviews, vol. 117, no. 5, pp. 4287-4341, 2017. 
[28] D. C. Hannah, G. Sai Gautam, P. Canepa, and G. Ceder, "On the balance of intercalation and conversion reactions in battery cathodes," Advanced Energy Materials, vol. 8, no. 20, p. 1800379, 2018.

[29] I. E. Castelli, T. Olsen, S. Datta, D. D. Landis, S. Dahl, K. S. Thygesen, and K. W. Jacobsen, "Computational screening of perovskite metal oxides for optimal solar light capture," Energy Environ. Sci., vol. 5, no. 2, pp. 5814-5819, 2012.

[30] D. C. Lonie and E. Zurek, "Identifying duplicate crystal structures: Xtalcomp, an open-source solution," Comput. Phys. Commun., vol. 183, no. 3, pp. 690-697, 2012.

[31] L. Beaulieu, K. Eberman, R. Turner, L. Krause, and J. Dahn, "Colossal reversible volume changes in lithium alloys," Electrochem. Solid-State Lett., vol. 4, no. 9, pp. A137-A140, 2001.

[32] Z. Rong, D. Kitchaev, P. Canepa, W. Huang, and G. Ceder, "An efficient algorithm for finding the minimum energy path for cation migration in ionic materials," The Journal of chemical physics, vol. 145, no. 7, p. 074112, 2016.

[33] O.-P. Koistinen, F. B. Dagbjartsdóttir, V. Ásgeirsson, A. Vehtari, and H. Jónsson, "Nudged elastic band calculations accelerated with gaussian process regression," The Journal of chemical physics, vol. 147, no. 15, p. 152720, 2017.

[34] J. A. G. Torres, P. C. Jennings, M. H. Hansen, J. R. Boes, and T. Bligaard, "Low-scaling algorithm for nudged elastic band calculations using a surrogate machine learning model," Physical review letters, vol. 122, no. 15, p. 156001, 2019.

[35] M. Liu, Z. Rong, R. Malik, P. Canepa, A. Jain, G. Ceder, and K. A. Persson, "Spinel compounds as multivalent battery cathodes: a systematic evaluation based on ab initio calculations," Energy Environ. Sci., vol. 8, no. 3, pp. 964-974, 2015.

[36] S. P. Ong, W. D. Richards, A. Jain, G. Hautier, M. Kocher, S. Cholia, D. Gunter, V. L. Chevrier, K. A. Persson, and G. Ceder, "Python materials genomics (pymatgen): A robust, open-source python library for materials analysis," Computational Materials Science, vol. 68, pp. 314-319, 2013.

[37] Z. Rong, R. Malik, P. Canepa, G. Sai Gautam, M. Liu, A. Jain, K. Persson, and G. Ceder, "Materials design rules for multivalent ion mobility in intercalation structures," Chemistry of Materials, vol. 27, no. 17, pp. 6016-6021, 2015.

[38] P. Canepa, S.-H. Bo, G. S. Gautam, B. Key, W. D. Richards, T. Shi, Y. Tian, Y. Wang, J. Li, and G. Ceder, "High magnesium mobility in ternary spinel chalcogenides," Nat. Commun., vol. 8, no. 1, p. $1759,2017$.

[39] I. Brown, "What factors determine cation coordination numbers?," Acta Crystallographica, Section B: Structural Science, vol. 44, no. 6, pp. 545-553, 1988.

[40] C. A. Marianetti, D. Morgan, and G. Ceder, "First-principles investigation of the cooperative jahnteller effect for octahedrally coordinated transition-metal ions," Phys. Rev. B, vol. 63, p. 224304, May 2001.

[41] X. Sun, P. Bonnick, V. Duffort, M. Liu, Z. Rong, K. A. Persson, G. Ceder, and L. F. Nazar, "A high capacity thiospinel cathode for mg batteries," Energy Environ. Sci., vol. 9, no. 7, pp. 2273-2277, 2016.

[42] "Supporting data - autonomous discovery of materials for intercalation electrodes." http://www.https://doi.org/10.11583/DTU.11627178.

[43] G. I. Csonka, J. P. Perdew, A. Ruzsinszky, P. H. Philipsen, S. Lebègue, J. Paier, O. A. Vydrov, and J. G. Ángyán, "Assessing the performance of recent density functionals for bulk solids," Phys. Rev. $B$, vol. 79, no. 15, p. 155107, 2009.

[44] L. Wang, T. Maxisch, and G. Ceder, "Oxidation energies of transition metal oxides within the gga+ u framework," Phys. Rev. B, vol. 73, no. 19, p. 195107, 2006.

[45] J. Rossmeisl, A. Logadottir, and J. K. Nørskov, "Electrolysis of water on (oxidized) metal surfaces," Chemical physics, vol. 319, no. 1-3, pp. 178-184, 2005.

[46] G. Henkelman, B. P. Uberuaga, and H. Jónsson, "A climbing image nudged elastic band method for finding saddle points and minimum energy paths," The Journal of chemical physics, vol. 113, no. 22, pp. 9901-9904, 2000.

[47] "Autonomous materials discovery (aimade)." http://www.aimade.org.

[48] K. Momma and F. Izumi, "Vesta 3 for three-dimensional visualization of crystal, volumetric and morphology data," J. Appl. Crystallogr., vol. 44, no. 6, pp. 1272-1276, 2011. 


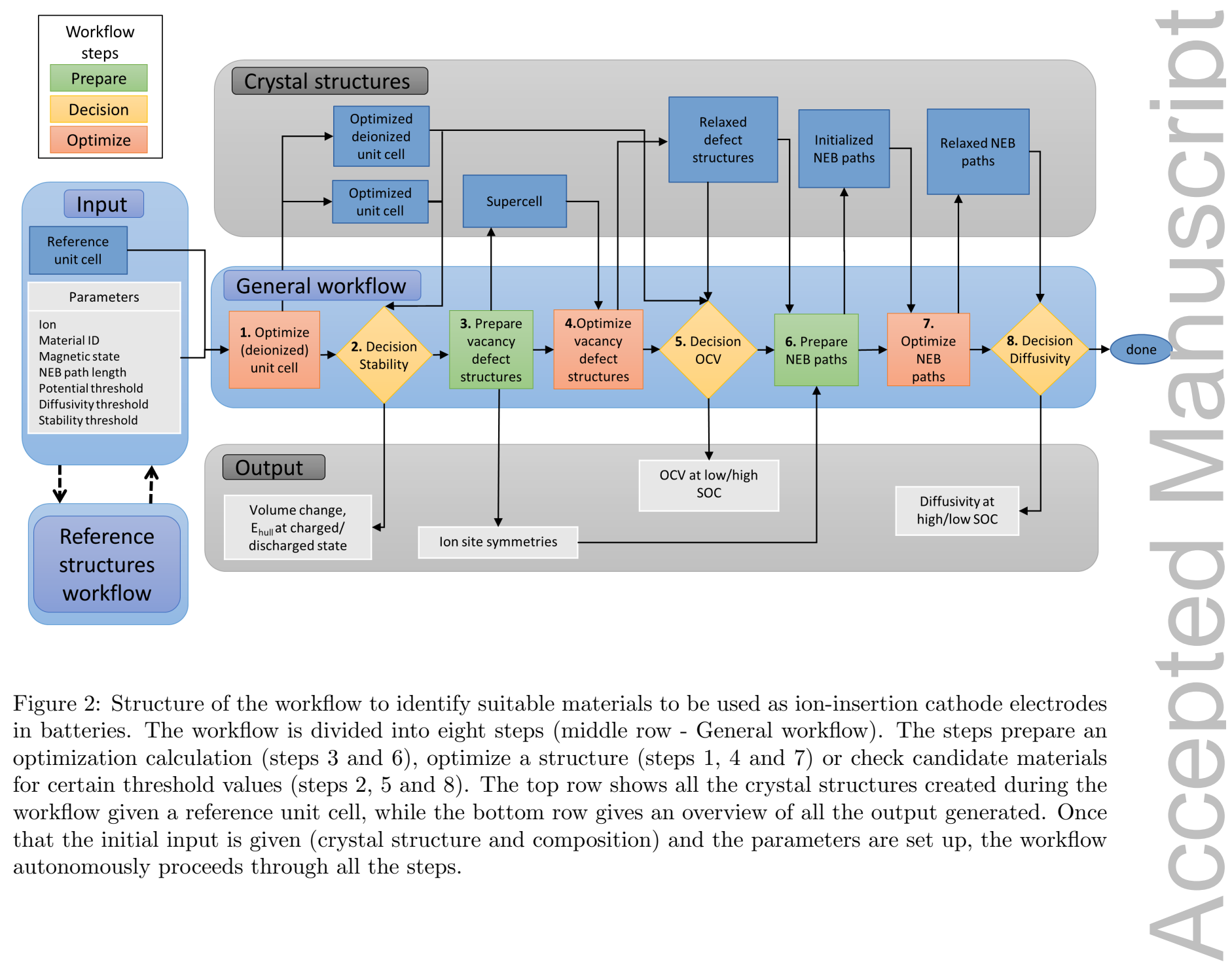




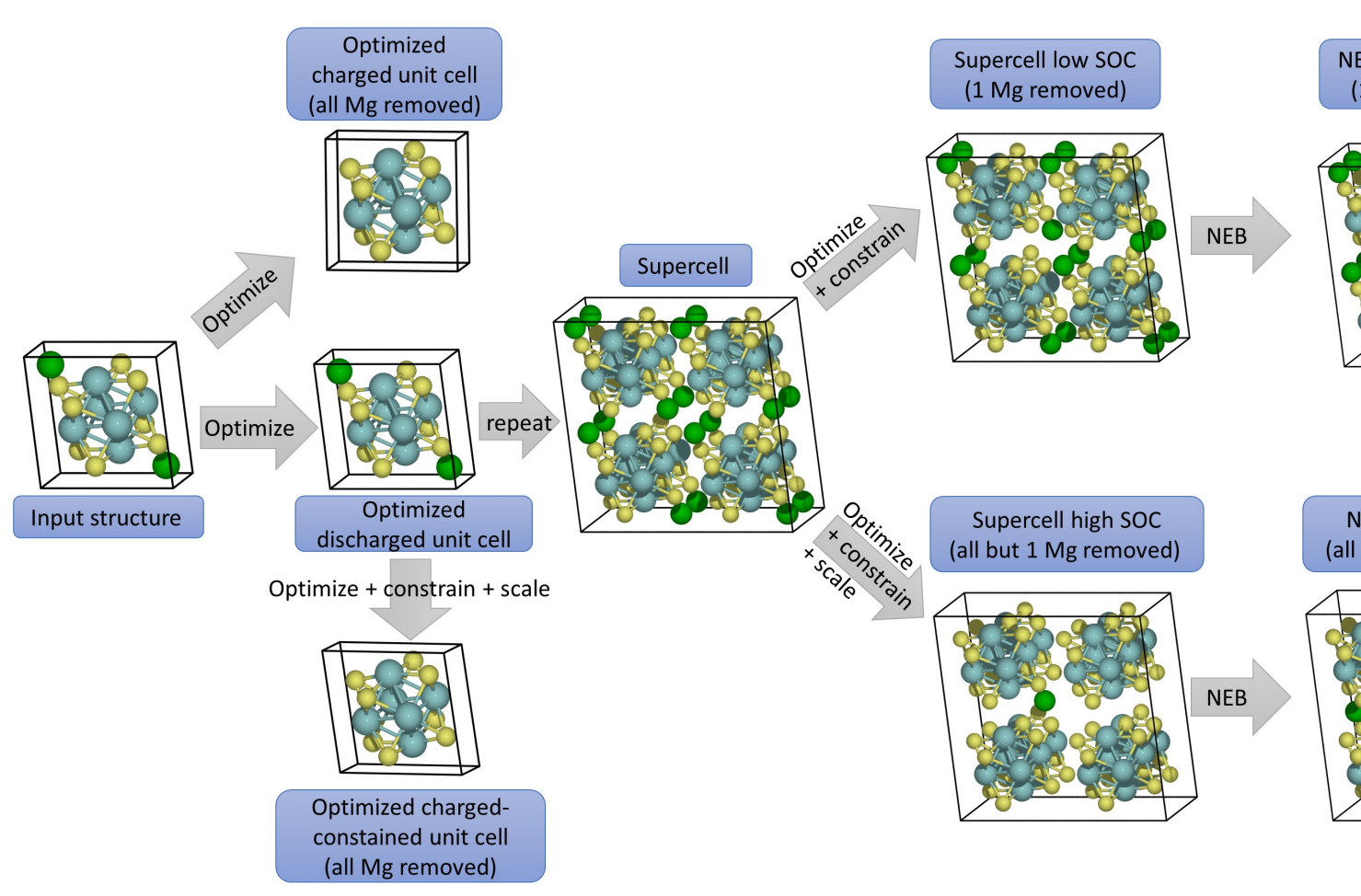

Figure 3: Structure creation throughout the workflow shown for the Chevrel Phase. The different labels on the arrows indicate how the resulting structure is obtained, i.e. optimize: Optimization of the cell shape, volume and atomic positions; optimize + constrain: Optimization of the atomic positions while keeping volume and cell shape unchanged; optimize + constrain + scale: Optimization of the atomic positions while re-scaling the volume according to the ratio $r=V_{\text {charged }} / V_{\text {discharged }}$ and keeping the cell shape fixed; repeat: solely repeating the cell; NEB: linear interpolation in between the initial and final site and subsequent optimization of the atomic positions while keeping cell shape and volume unchanged. Color coding: dark green (Magnesium), yellow (Sulfur), light blue (Molybdenum). The grey shaded spheres in the NEB path structures indicate the linear initialization of the NEB path and therefore the intermediate images. Both, initial and final site, are shown as dark green spheres.

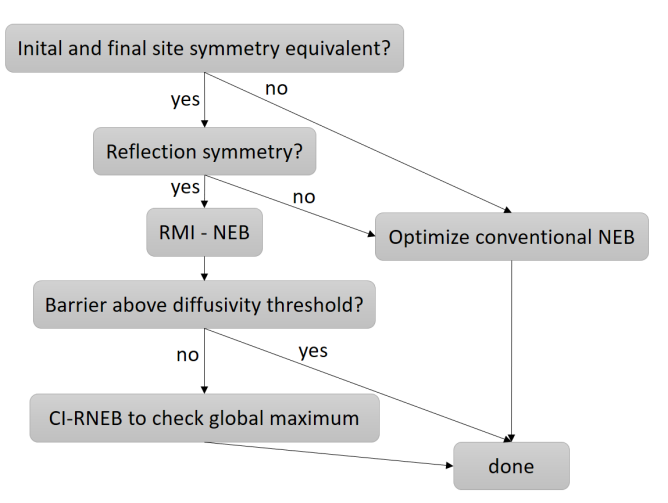

(a) NEB workflow

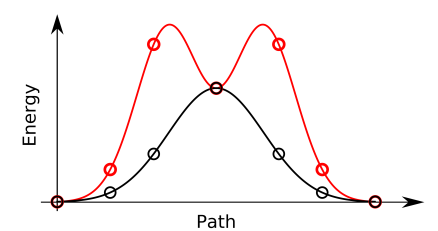

(b) Conventional NEB

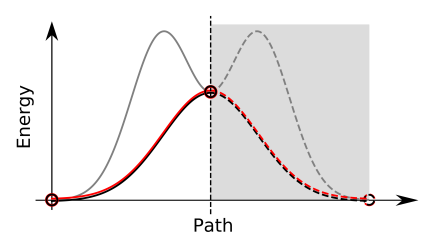

(d) RMI-NEB

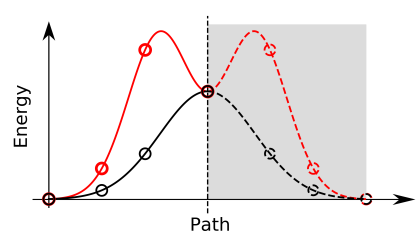

(c) R-NEB

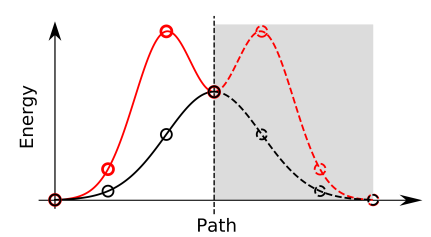

(e) CI-RNEB

Figure 4: (a) Logic for the preparation of in-equivalent NEB paths by including reflection symmetry considerations, with the goal of reducing the computational time needed to find the transition state. The four figures to the right sketch the principles of the different ways of running a NEB calculation including a conventional NEB (b), reflective NEB or R-NEB (c), reflective-middle image NEB or RMI-NEB (d) and the climbing image reflective NEB or CI-RNEB (e). The images that lay in the grey shaded area indicate that these images are not calculated explicitly but are created by using symmetry operations. This is done by running a recent implementation of the NEB method [17]. The two colored lines show an example of a truly bellshaped energy barrier (black solid line) as well as an energy barrier with two maxima (red solid line). 


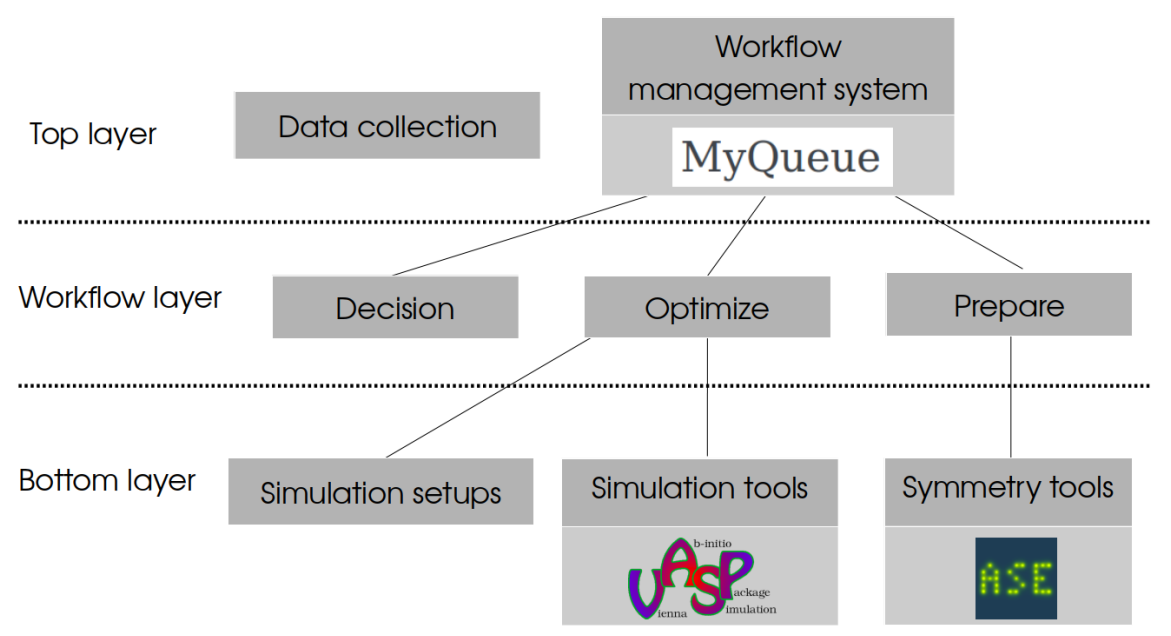

Figure 5: Different layers of the workflow.
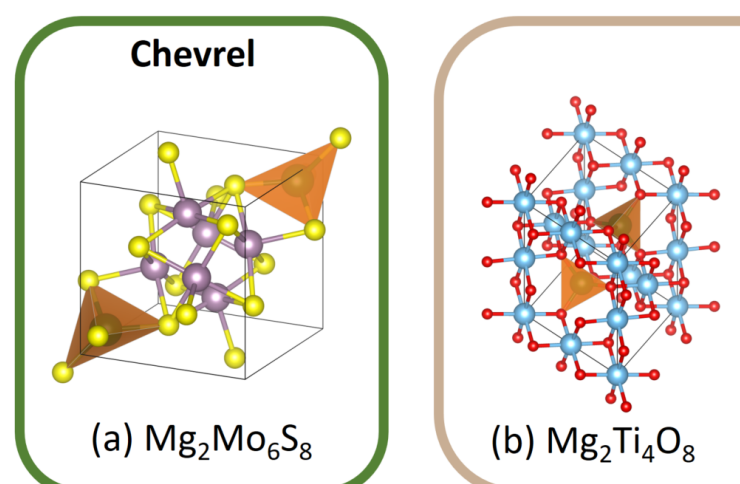

(b) $\mathrm{Mg}_{2} \mathrm{Ti}_{4} \mathrm{O}_{8}$

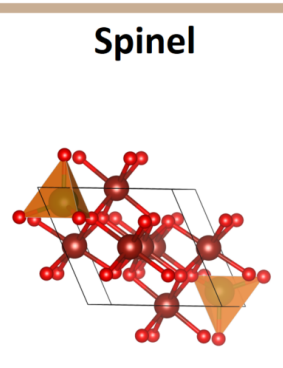

(c) $\mathrm{Mg}_{2} \mathrm{~V}_{4} \mathrm{O}_{8}$

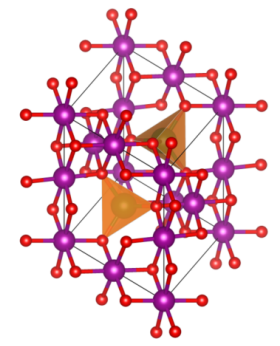

(d) $\mathrm{Mg}_{2} \mathrm{Mn}_{4} \mathrm{O}_{8}$

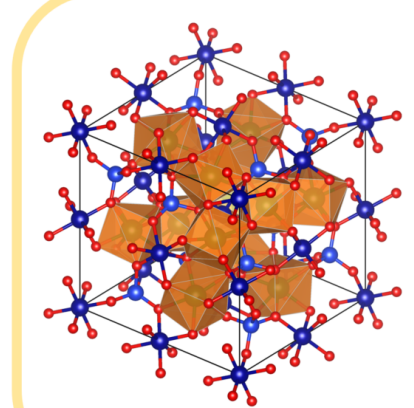

(e) $\mathrm{Mg}_{12} \mathrm{Cr}_{8}\left(\mathrm{SiO}_{4}\right)_{12}$

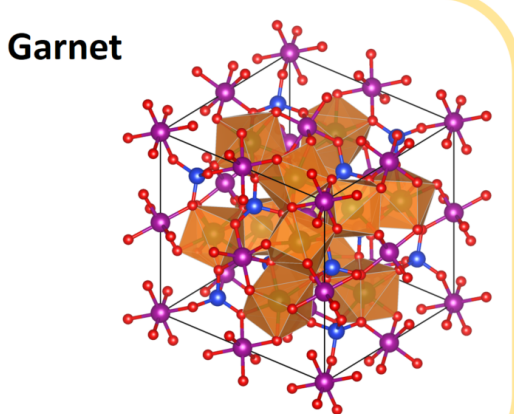

(f) $\mathrm{Mg}_{12} \mathrm{Mn}_{8}\left(\mathrm{SiO}_{4}\right)_{12}$

Figure 6: Unit cells of the six candidates investigated. The $\mathrm{Mg}^{2+}$ ions are inside the polyhedron to visualize differences in coordination. Shown are the Chevrel Phase, $\mathrm{MgMo}_{3} \mathrm{~S}_{4}$ (a, Materials Project-ID: mp-677217); Spinel structures, $\mathrm{MgB}_{2} \mathrm{O}_{4}$ with $\mathrm{B}=\{\mathrm{Ti}, \mathrm{V}, \mathrm{Mn}\}$ (b: ICSD-65729, c: ICSD-60412, d: ICSD-290600); and Garnet structures, $\mathrm{Mg}_{3} \mathrm{~B}_{2}\left(\mathrm{SiO}_{4}\right)_{3}$ with $\mathrm{B}=\{\mathrm{Cr}, \mathrm{Mn}\}$ (e: ICSD-77432 , f: ICSD-27374). The $\mathrm{Mg}^{2+}$ ion occupies a tetrahedral site in structures (a-d). In the Garnet structures (e,f) $\mathrm{Mg}^{2+}$ has eight closest oxygen neighbors. The structures are visualized using VESTA. [48] 


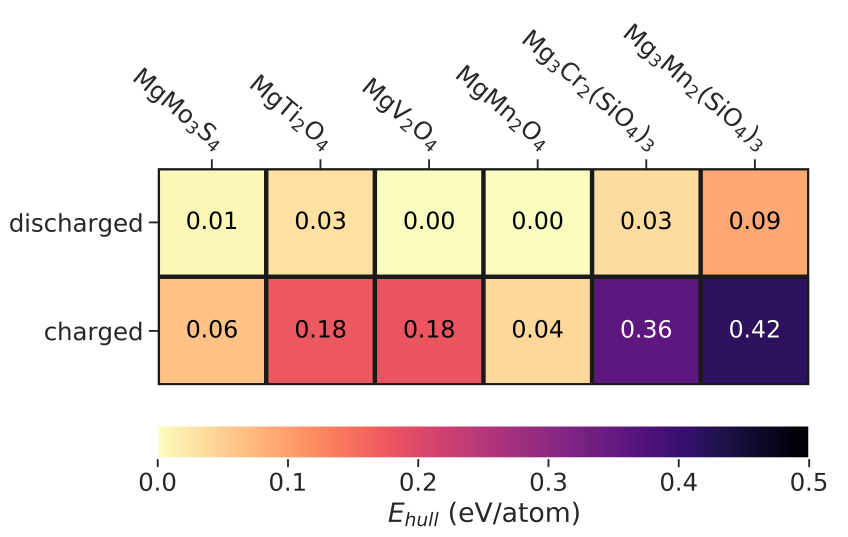

Figure 7: Thermodynamic stability of the charged and discharged phases. Reference structures for calculating the ground state hulls have been taken from the Materials Project database. [24]

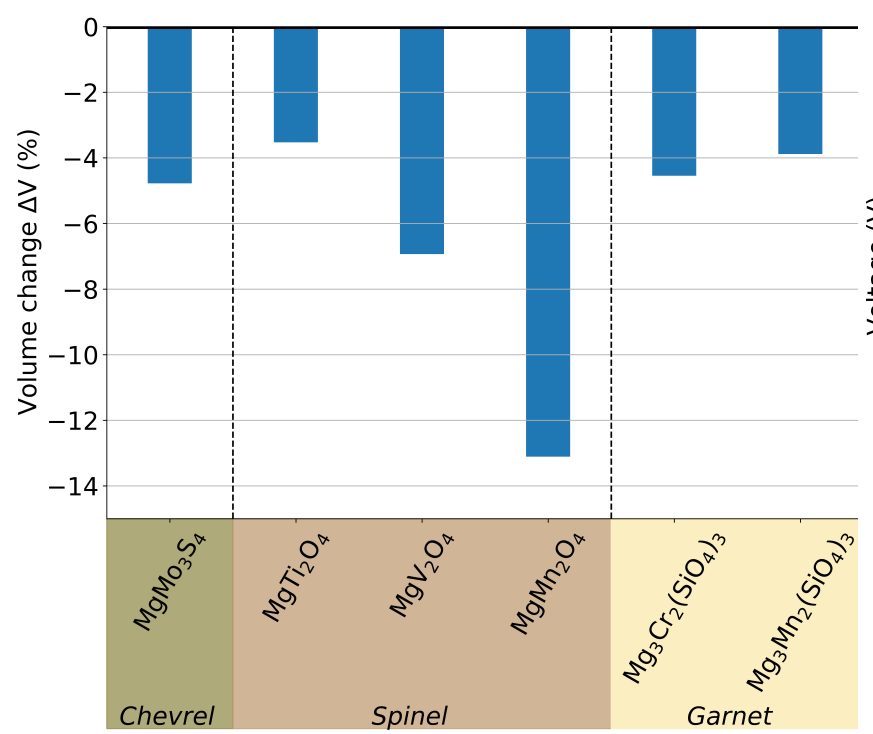

(a)

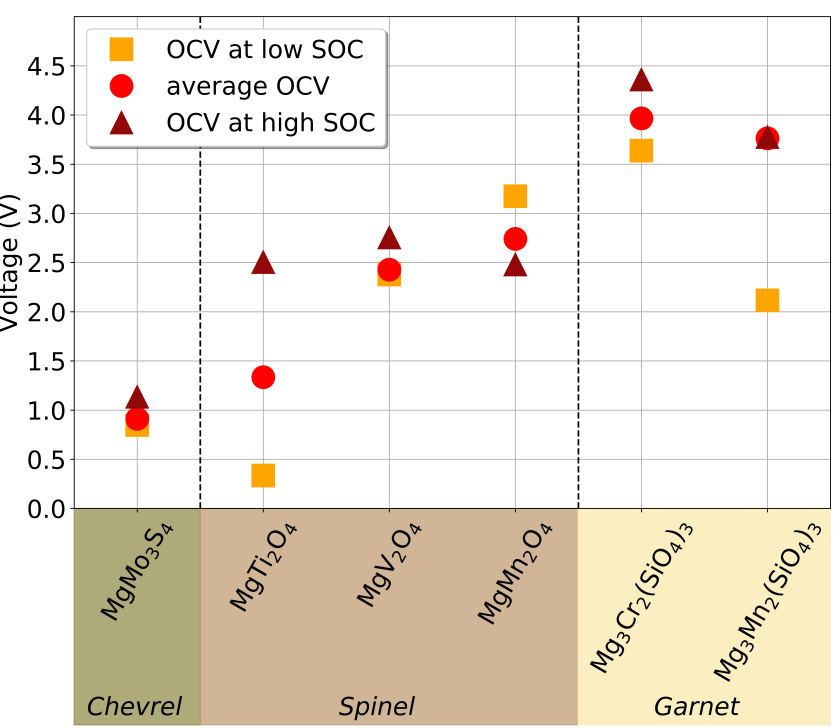

(b)

Figure 8: (a) Volume changes (in \%) upon charging the cathode material as calculated using Eq. (1). (b) OCVs at different charge states calculated according to Eqs. (2), (3) and (4). 


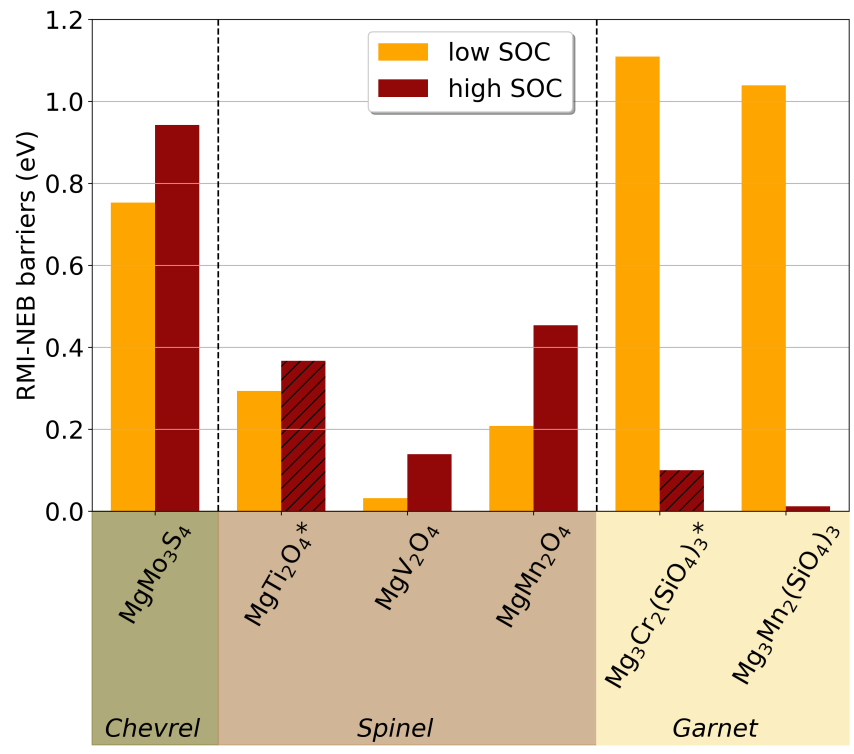

(a)

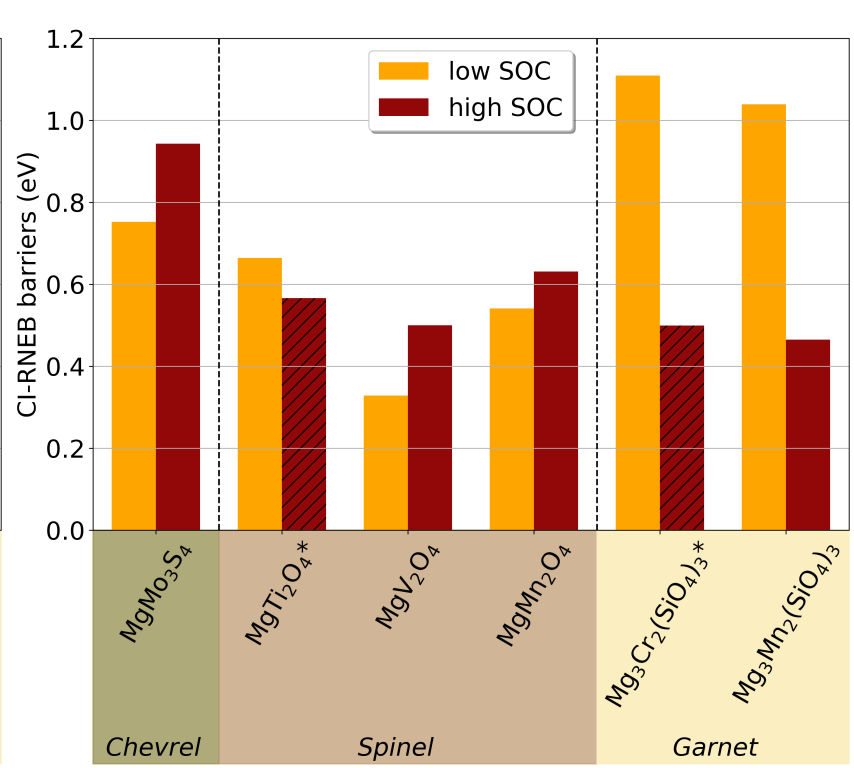

(b)

Figure 9: Height of the diffusion barrier calculated using the RMI-NEB/CI-RNEB method. Only a single intermediate image (middle image) is used to determine the transition state for the RMI-NEB method. The CI-RNEB path consists of five intermediate images including the reflected and middle images. The hatched bar for materials marked with an asterisk $(*)$ indicates that the middle image is lower in energy than the initial and final state. 